\title{
Detailed error analysis for a fractional Adams method with graded meshes
}

\author{
Yanzhi Liu' ${ }^{2}$ Jason Roberts ${ }^{1} \cdot$ Yubin Yan $^{1}$
}

Received: 15 March 2017 / Accepted: 12 September 2017 / Published online: 21 September 2017 (C) The Author(s) 2017. This article is an open access publication

Abstract We consider a fractional Adams method for solving the nonlinear fractional differential equation ${ }_{0}^{C} D_{t}^{\alpha} y(t)=f(t, y(t)), \alpha>0$, equipped with the initial conditions $y^{(k)}(0)=y_{0}^{(k)}, k=0,1, \ldots,\lceil\alpha\rceil-1$. Here, $\alpha$ may be an arbitrary positive number and $\lceil\alpha\rceil$ denotes the smallest integer no less than $\alpha$ and the differential operator is the Caputo derivative. Under the assumption ${ }_{0}^{C} D_{t}^{\alpha} y \in C^{2}[0, T]$, Diethelm et al. (Numer. Algor. 36, 31-52, 2004) introduced a fractional Adams method with the uniform meshes $t_{n}=T(n / N), n=0,1,2, \ldots, N$ and proved that this method has the optimal convergence order uniformly in $t_{n}$, that is $O\left(N^{-2}\right)$ if $\alpha>1$ and $O\left(N^{-1-\alpha}\right)$ if $\alpha \leq 1$. They also showed that if ${ }_{0}^{C} D_{t}^{\alpha} y(t) \notin C^{2}[0, T]$, the optimal convergence order of this method cannot be obtained with the uniform meshes. However, it is well-known that for $y \in C^{m}[0, T]$ for some $m \in \mathbb{N}$ and $0<\alpha<m$, the Caputo fractional derivative ${ }_{0}^{C} D_{t}^{\alpha} y(t)$ takes the form " ${ }_{0}^{C} D_{t}^{\alpha} y(t)=c t^{\lceil\alpha\rceil-\alpha}+$ smoother terms" (Diethelm et al. Numer. Algor. 36, 31-52, 2004), which implies that ${ }_{0}^{C} D_{t}^{\alpha} y$ behaves as $t^{\lceil\alpha\rceil-\alpha}$ which is not in $C^{2}[0, T]$. By using the graded meshes $t_{n}=T(n / N)^{r}, n=$ $0,1,2, \ldots, N$ with some suitable $r>1$, we show that the optimal convergence order of this method can be recovered uniformly in $t_{n}$ even if ${ }_{0}^{C} D_{t}^{\alpha} y$ behaves as

Yubin Yan

y.yan@chester.ac.uk

Yanzhi Liu

115027431@qq.com

Jason Roberts

j.roberts@chester.ac.uk

1 Department of Mathematics, University of Chester, Thornton Science Park, Pool Lane, Ince, $\mathrm{CH} 2$ 4NU, UK

2 Department of Mathematics, Lvliang University, Lüliang, People's Republic of China 
$t^{\sigma}, 0<\sigma<1$. Numerical examples are given to show that the numerical results are consistent with the theoretical results.

Keywords Fractional differential equations $\cdot$ Caputo derivative $\cdot$ Adams method

Mathematics subject classification (2010) 65L06 - 26A33 - 65B05 - 65L05 · $65 \mathrm{~L} 20 \cdot 65 \mathrm{R} 20$

\section{Introduction}

In this paper, we will consider a numerical method for solving the following fractional nonlinear differential equation, with $\alpha>0$,

$$
{ }_{0}^{C} D_{t}^{\alpha} y(t)=f(t, y(t)), t>0, y^{(k)}(0)=y_{0}^{(k)}, k=0,1, \ldots,\lceil\alpha\rceil-1,
$$

where the $y_{0}^{(k)}$ may be arbitrary real numbers and ${ }_{0}^{C} D_{t}^{\alpha} y(t)$ denotes the Caputo fractional derivative defined by

$$
{ }_{0}^{C} D_{t}^{\alpha} y(t)=\frac{1}{\Gamma(\lceil\alpha\rceil-\alpha)} \int_{0}^{t}(t-s)^{\lceil\alpha\rceil-\alpha-1} y^{\lceil\alpha\rceil}(s) d s,
$$

where $\lceil\alpha\rceil$ is the smallest integer $\geq \alpha$. As usual we demand that the function $f$ is continuous and fulfills a Lipschitz condition with respect to its second argument with Lipschitz constant $L$ on a suitable set $G$. Under these assumptions, Diethelm et al. [7, Theorems 2.1, 2.2] showed that (1.1) has a unique solution $y$ on some interval $[0, T]$.

It is well-known that (1.1) is equivalent to [7, Lemma 2.3]

$$
y(t)=\sum_{\nu=0}^{\lceil\alpha\rceil-1} y_{0}^{(\nu)} \frac{t^{\nu}}{\nu !}+\frac{1}{\Gamma(\alpha)} \int_{0}^{t}(t-s)^{\alpha-1} f(s, y(s)) d s .
$$

Equations of this type arise in a number of applications where models based on fractional calculus are used, such as viscoelastic materials, anomalous diffusion, signal processing, and control theory; see Oldham and Spanier [16], Kilbas et al. [10], Podlubny [20].

The analytic solution of (1.1) for the general function $f$ is not known. Therefore, we have to apply some numerical methods for solving (1.1). Stability and convergence of such numerical methods are analyzed under certain smoothness assumptions for the solutions of (1.1); see, for example, [1, 2, 7, 11, 14, 17, 18, 23, 26].

Most analysis of the numerical methods for solving (1.1) is deduced under the assumptions that the meshes are uniform; see, for example, [7-9, 13, 14, 26]. To obtain a higher order numerical method with uniform meshes, the solutions or data of (1.1) are required to be sufficiently smooth, for example, ${ }_{0}^{C} D_{t}^{\alpha} y \in C^{m}[0, T], m \geq 2$ in [8, Theorem 3.2]. However, as we will see below in Theorem 1.2, although $y \in C^{m}[0, T]$ for some $m \in \mathbb{N}, 0<\alpha<m$, the Caputo fractional derivative ${ }_{0}^{C} D_{t}^{\alpha} y$ behaves as $t^{\lceil\alpha\rceil-\alpha}$ when $y^{\lceil\alpha\rceil}(0) \neq 0, \alpha>0$. Therefore, it is interesting to design some numerical methods which have the optimal convergence orders when ${ }_{0}^{C} D_{t}^{\alpha} y$ 
behaves as $t^{\lceil\alpha\rceil-\alpha}, \alpha>0$. Diethelm [4, Theorem 3.1] used the graded meshes to recover the optimal convergence order for the approximation of the Hadamard finitepart integral. Recently, Stynes et al. [21,22] applied the graded meshes to recover the convergence order of the finite difference method for solving a time-fractional diffusion equation when the solution is not sufficiently smooth. This excellent approach in $[21,22]$ allows to obtain a (relatively) high convergence order without the otherwise required very unnatural smoothness assumptions on the given solution. Other works for solving fractional differential equations with nonuniform meshes may be found in, for example, [12, 19, 24, 25].

Motivated by the ideas in Diethelm [4] and Stynes et al. [22], we will introduce a numerical method for solving (1.1) with the graded meshes and we prove that the optimal convergence order uniformly in $t_{n}$ for the proposed numerical method can be recovered when ${ }_{0}^{C} D_{t}^{\alpha} y(t), \alpha>0$ behaves as $t^{\sigma}, 0<\sigma<1$.

Before we introduce our numerical method, we recall some well-known smoothness properties of the solution $y$ of (1.1) under some assumptions of $f$.

Theorem 1.1 [15, Lubich, 1983, Theorem 2.1]

1. Let $\alpha>0$. Assume that $f \in C^{2}(G)$. Define $\hat{v}:=\left\lceil\frac{1}{\alpha}\right\rceil-1$. Then there exist a function $\psi \in C^{1}[0, T]$ and some $c_{1}, c_{2}, \ldots, c_{\hat{v}} \in \mathbb{R}$ such that the solution $y$ of (1.1) can be expressed in the form

$$
y(t)=\psi(t)+c_{1} t^{\alpha}+c_{2} t^{2 \alpha}+c_{3} t^{3 \alpha}+\cdots+c_{\hat{v}} t^{\hat{\nu} \alpha} .
$$

2. Let $\alpha>0$. Assume that $f \in C^{3}(G)$. Define $\hat{v}:=\left\lceil\frac{2}{\alpha}\right\rceil-1$ and $\tilde{v}:=\left\lceil\frac{1}{\alpha}\right\rceil-1$. Then there exist a function $\psi \in C^{2}[0, T]$ and some $c_{1}, c_{2}, \ldots, c_{\hat{v}} \in \mathbb{R}$ and $d_{1}, d_{2}, \ldots, d_{\tilde{v}} \in \mathbb{R}$ such that the solution $y$ of (1.1) can be expressed in the form

$$
y(t)=\psi(t)+\sum_{\nu=1}^{\hat{v}} c_{\nu} t^{\nu \alpha}+\sum_{\nu=1}^{\tilde{v}} d_{\nu} t^{1+\nu \alpha} .
$$

For example, when $0<\alpha<1, f \in C^{2}(G)$, we have $\hat{v}=\left\lceil\frac{1}{\alpha}\right\rceil-1 \geq 1$ and

$$
y=c t^{\alpha}+\text { smoother terms, }
$$

which implies that the solution $y$ of (1.1) behaves as $t^{\alpha}, 0<\alpha<1$ when $f \in C^{2}(G)$.

Theorem 1.2 [8, Theorem 2.2] If $y \in C^{m}[0, T]$ for some $m \in \mathbb{N}$ and $0<\alpha<m$, then

$$
{ }_{0}^{C} D_{t}^{\alpha} y(t)=\varphi(t)+\sum_{l=0}^{m-\lceil\alpha\rceil-1} \frac{y^{(l+\lceil\alpha\rceil)}(0)}{\Gamma(\lceil\alpha\rceil-\alpha+l+1)} t^{\lceil\alpha\rceil-\alpha+l},
$$

with some function $\varphi \in C^{m-\lceil\alpha\rceil}[0, T]$. Moreover, the $(m-\lceil\alpha\rceil)$ th derivative of $\varphi$ satisfies a Lipschitz condition of order $\lceil\alpha\rceil-\alpha$.

For example, when $0<\alpha<1, y \in C^{m}[0, T], m \geq 2$, we have

$$
{ }_{0}^{C} D_{t}^{\alpha} y(t)=\varphi(t)+\frac{y^{\prime}(0)}{\Gamma(2-\alpha)} t^{1-\alpha}+\text { smoother terms, }
$$


where $\varphi \in C^{m-1}[0, T]$ which implies that the Caputo fractional derivative ${ }_{0}^{C} D_{t}^{\alpha} y(t), 0<\alpha<1$ behaves as $t^{1-\alpha}$ when $y^{\prime}(0) \neq 0$. Similarly, when $1<\alpha<$ 2, $y \in C^{m}[0, T], m \geq 3$, we have

$$
{ }_{0}^{C} D_{t}^{\alpha} y(t)=\varphi(t)+\frac{y^{\prime \prime}(0)}{\Gamma(3-\alpha)} t^{2-\alpha}+\text { smoother terms, }
$$

where $\varphi \in C^{m-2}[0, T]$.

In view of Theorems 1.1 and 1.2, we see that smoothness of one of the functions $y$ and ${ }_{0}^{C} D_{t}^{\alpha} y$ will imply nonsmoothness of the other unless some special conditions are fulfilled. Based on Theorems 1.1 and 1.2, we introduce the following assumption. The similar assumption for the smoothness of the solution $u$ of the time-fractional diffusion equation is introduced in Stynes et al. [22, Theorem 2.1].

Assumption 1 Let $0<\sigma<1$ and let $g:={ }_{0}^{C} D_{t}^{\alpha} y \in C^{2}(0, T]$ with $\alpha>0$. There exists a constant $c>0$ such that

$$
\left|g^{\prime}(t)\right| \leq c t^{\sigma-1}, \quad\left|g^{\prime \prime}(t)\right| \leq c t^{\sigma-2}
$$

Remark 1.3 The condition (1.4) gives the behavior of $g(t)$ near $t=0$ and implies that $g(t)$ has the singularity near $t=0$. It is obvious that $g \notin C^{2}[0, T]$. For example, we may choose $g(t)=t^{\sigma}$ with $0<\sigma<1$.

Let $N$ be a positive integer and let $0=t_{0}<t_{1}<\cdots<t_{N}=T$ be the graded meshes on $[0, T]$ defined by

$$
t_{j}=T(j / N)^{r}, j=0,1,2, \ldots, N, \quad \text { with } r \geq 1 .
$$

For simplicity, we assume that $T=1$ in this paper.

Let us now introduce the fractional Adams method with the graded meshes (1.5). Such methods with the uniform meshes have been introduced and analyzed in Diethelm [5, Appendix C] in the general case and Diethelm et al. [8].

Denote $y_{j} \approx y\left(t_{j}\right), j=0,1,2, \ldots, n+1$ with $n=0,1,2, \ldots, N-1$, the approximation of $y\left(t_{j}\right)$, we define the following predictor-corrector Adams method for solving (1.3), with $\alpha>0$ :

$$
\begin{aligned}
& y_{n+1}^{P}=\sum_{\nu=0}^{\lceil\alpha\rceil-1} y_{0}^{(\nu)} \frac{t_{n+1}}{\nu !}+\frac{1}{\Gamma(\alpha)} \sum_{j=0}^{n} b_{j, n+1} f\left(t_{j}, y_{j}\right), \\
& y_{n+1}=\sum_{\nu=0}^{\lceil\alpha\rceil-1} y_{0}^{(\nu)} \frac{t_{n+1}}{\nu !}+\frac{1}{\Gamma(\alpha)}\left(\sum_{j=0}^{n} a_{j, n+1} f\left(t_{j}, y_{j}\right)+a_{n+1, n+1} f\left(t_{n+1}, y_{n+1}^{P}\right)\right), \\
& y_{0}^{(\nu)} \text { is given, }
\end{aligned}
$$

where the weights $b_{j, n+1}, j=0,1,2, \ldots, n$ satisfy

$$
b_{j, n+1}=\frac{N^{-r \alpha}}{\alpha}\left(\left((n+1)^{r}-j^{r}\right)^{\alpha}-\left((n+1)^{r}-(j+1)^{r}\right)^{\alpha}\right) \text {, }
$$


and the weights $a_{j, n+1}, j=0,1,2, \ldots, n+1$ satisfy

$$
\begin{aligned}
a_{0, n+1}= & \frac{N^{-r \alpha}}{\alpha(1+\alpha)}\left((n+1)^{r \alpha}(\alpha+1)+\left((n+1)^{r}-1\right)^{\alpha+1}-(n+1)^{r(\alpha+1)}\right), \\
a_{j, n+1}= & \frac{N^{-r \alpha}}{\alpha(1+\alpha)}\left(\frac{\left[(n+1)^{r}-(j-1)^{r}\right]^{\alpha+1}-\left[(n+1)^{r}-j^{r}\right]^{\alpha+1}}{j^{r}-(j-1)^{r}}\right. \\
& \left.+\frac{\left[(n+1)^{r}-(j+1)^{r}\right]^{\alpha+1}-\left[(n+1)^{r}-j^{r}\right]^{\alpha+1}}{(j+1)^{r}-j^{r}}\right), j=1,2, \ldots, n, \\
a_{n+1, n+1}= & \frac{N^{-r \alpha}}{\alpha(1+\alpha)}\left((n+1)^{r}-n^{r}\right)^{\alpha} .
\end{aligned}
$$

The predictor term $y_{n+1}^{P}$ in (1.6) is obtained by approximating the integral $\int_{0}^{t_{n+1}}\left(t_{n+1}-s\right)^{\alpha-1} f(s, y(s)) d s$ in (1.3) with $\int_{0}^{t_{n+1}}\left(t_{n+1}-s\right)^{\alpha-1} P_{0}(s) d s$, where $P_{0}(s)$ is the piecewise constant function defined on $\left[0, t_{n+1}\right]$, i.e.,

$$
P_{0}(s)=f\left(t_{j}, y\left(t_{j}\right)\right), s \in\left[t_{j}, t_{j+1}\right], j=0,1,2, \ldots, n .
$$

Similarly, the corrector term $y_{n+1}$ in (1.6) is obtained by approximating the integral $\int_{0}^{t_{n+1}}\left(t_{n+1}-s\right)^{\alpha-1} f(s, y(s)) d s$ in (1.3) with $\int_{0}^{t_{n+1}}\left(t_{n+1}-s\right)^{\alpha-1} P_{1}(s) d s$, where $P_{1}(s)$ is the piecewise linear function defined on $\left[0, t_{n+1}\right]$, i.e.,

$P_{1}(s)=\frac{s-t_{j+1}}{t_{j}-t_{j+1}} f\left(t_{j}, y\left(t_{j}\right)\right)+\frac{s-t_{j}}{t_{j+1}-t_{j}} f\left(t_{j+1}, y\left(t_{j+1}\right)\right), s \in\left[t_{j}, t_{j+1}\right], j=0,1,2, \ldots, n$.

We remark that when $r=1$, the weights in (1.8) reduce to the weights in Diethelm et al. [8, (1.14)] with the uniform meshes.

Under the assumption that $g(t):={ }_{0}^{C} D_{t}^{\alpha} y(t) \in C^{2}[0, T]$ and $r=1$ (i.e., uniform meshes), Diethelm et al. [8] proved the following error estimates, i.e., [8, Theorem 3.2]:

Theorem 1.4 Let $\alpha>0$ and assume that $g:={ }_{0}^{C} D_{t}^{\alpha} y \in C^{2}[0, T]$ for some suitable $T$. Assume that $y\left(t_{j}\right)$ and $y_{j}$ are the solutions of (1.3) and (1.6), respectively. Let $r=1$ (uniform meshes). Then

$$
\max _{0 \leq j \leq N}\left|y\left(t_{j}\right)-y_{j}\right| \leq\left\{\begin{array}{cc}
C N^{-(1+\alpha)}, & \text { if } 0<\alpha \leq 1, \\
C N^{-2}, & \text { if } \alpha>1 .
\end{array}\right.
$$

In this work, under the Assumption 1, and $r>1$, we shall prove the following error estimates:

Theorem 1.5 Let $\alpha>0$ and assume that $g:={ }_{0}^{C} D_{t}^{\alpha} y$ satisfies Assumption 1.

1. If $0<\alpha \leq 1$, assume that $y\left(t_{j}\right)$ and $y_{j}$ are the solutions of (1.3) and (1.6), respectively, then we have

$$
\max _{0 \leq j \leq N}\left|y\left(t_{j}\right)-y_{j}\right| \leq\left\{\begin{array}{cl}
C N^{-r(\sigma+\alpha)}, & \text { if } r(\alpha+\sigma)<1+\alpha, \\
C N^{-r(\sigma+\alpha)} \ln (N), & \text { if } r(\alpha+\sigma)=1+\alpha, \\
C N^{-(1+\alpha)}, & \text { if } r(\alpha+\sigma)>1+\alpha .
\end{array}\right.
$$

2. If $\alpha>1$, then we have

$$
\max _{0 \leq j \leq N}\left|y\left(t_{j}\right)-y_{j}\right| \leq\left\{\begin{array}{cc}
C N^{-r(1+\sigma)}, & \text { if } r(1+\sigma)<2 \\
C N^{-2} \ln (N), & \text { if } r(1+\sigma)=2 \\
C N^{-2}, & \text { if } r(1+\sigma)>2
\end{array}\right.
$$


Remark 1.6 By Theorem 1.1, assume that $f \in C^{m}(G), m \geq 2$ and $\alpha \in(0,1)$, then, with some constants $c_{1}, c_{2}, \ldots, c_{\hat{v}} \in \mathbb{R}$,

$$
y=c_{1} t^{\alpha}+c_{2} t^{2 \alpha}+\cdots+c_{\hat{\nu}} t^{\hat{\nu} \alpha}+\text { smoother terms, }
$$

which implies that, with some constants $d_{1}, d_{2}, \ldots, d_{\hat{v}} \in \mathbb{R}$,

$$
\begin{aligned}
g: & ={ }_{0}^{C} D_{t}^{\alpha} y=d_{1} t^{\alpha-\alpha}+d_{2} t^{2 \alpha-\alpha}+\cdots+d_{\hat{v}} t^{\hat{v} \alpha-\alpha}+\text { smoother terms } \\
& =d_{1}+d_{2} t^{\alpha}+\cdots+d_{\hat{v}} t^{(\hat{v}-1) \alpha}+\text { smoother terms. }
\end{aligned}
$$

We see $g:={ }_{0}^{C} D_{t}^{\alpha} y$ behaves as $c+c t^{\alpha}$; therefore, we may apply Theorem 1.4 with $\sigma=\alpha$ in this case.

Remark 1.7 If one uses $M>1$ corrector iterations instead of just one, under the assumption that $g:={ }_{0}^{C} D_{t}^{\alpha} y$ is sufficiently smooth, Diethelm [6] showed that the order in Theorem 1.4 with the uniform meshes can be improved to $O\left(N^{-\min \{2,1+M \alpha\}}\right)$ for $\alpha>0$. For the graded meshes, we may show that the order in Theorem 1.4 can also be improved to $O\left(N^{-\min \{2,1+M \alpha\}}\right)$ by choosing $r>1$ such that $r(M \alpha+\sigma)>\min \{2,1+M \alpha\}$ when $0<\alpha \leq 1$. For $\alpha>1$, it is not necessary to use $M>1$ correctors since we can always get $O\left(N^{-2}\right)$ when we choose $r$ such that $r(1+\sigma)>2$ for any $M \geq 1$ correctors.

Remark 1.8 The modification of the basic Adams-Bashforth-Moulton method suggested by Deng [3] for the case of a uniform grid can be applied for the graded mesh used in this paper as well. This should lead to a reduction of the computational cost without an increased error.

We remark that the optimal convergence order $O\left(N^{-\min (1+\alpha, 2)}\right), \alpha>0$ obtained in Theorem 1.3 for the numerical method (1.6) for the smooth $g$ with the uniform meshes with $r=1$ can be recovered in Theorem 1.4 for the nonsmooth $g$ with the graded meshes (1.5) with $r>1$.

The paper is organized as follows. In Section 1. we introduce the predictorcorrector method for solving (1.1) with the graded meshes. In Section 2, we prove our main result Theorem 1.4. Finally in Section 3, we give some numerical examples which show that the numerical results are consistent with the theoretical results.

Throughout, the notations $C$ and $c$, with or without a subscript, denote generic constants, which may differ at different occurrences, but are always independent of the mesh size.

\section{Proof of theorem 1.4}

In this section, we will give the proof of Theorem 1.4. To do this, we need some preliminary lemmas.

Lemma 2.1 Let $\alpha>0$. Assume that $g$ satisfies Assumption 1 . 
1. If $0<\alpha \leq 1$, then

$$
\left|\int_{0}^{t_{n+1}}\left(t_{n+1}-s\right)^{\alpha-1}\left(g(s)-P_{1}(s)\right) d s\right| \leq\left\{\begin{array}{cl}
C N^{-r}(\alpha+\sigma), & \text { if } r(\alpha+\sigma)<2, \\
C N^{-2} \ln (N), & \text { if } r(\alpha+\sigma)=2, \\
C N^{-2}, & \text { if } r(\alpha+\sigma)>2 .
\end{array}\right.
$$

2. If $\alpha>1$, then

$$
\left|\int_{0}^{t_{n+1}}\left(t_{n+1}-s\right)^{\alpha-1}\left(g(s)-P_{1}(s)\right) d s\right| \leq\left\{\begin{array}{cl}
C N^{-r}(1+\sigma), & \text { if } r(1+\sigma)<2, \\
C N^{-2} \ln (N), & \text { if } r(1+\sigma)=2, \\
C N^{-2}, & \text { if } r(1+\sigma)>2,
\end{array}\right.
$$

where $P_{1}(s)$ is the piecewise linear function defined by, with $j=0,1,2, \ldots, n$,

$$
P_{1}(s)=\frac{s-t_{j+1}}{t_{j}-t_{j+1}} g\left(t_{j}\right)+\frac{s-t_{j}}{t_{j+1}-t_{j}} g\left(t_{j+1}\right), \quad s \in\left[t_{j}, t_{j+1}\right] .
$$

Proof Note that, with $n=0,1,2, \ldots, N-1$,

$$
\begin{aligned}
& \int_{0}^{t_{n+1}}\left(t_{n+1}-s\right)^{\alpha-1}\left(g(s)-P_{1}(s)\right) d s \\
& =\left(\int_{0}^{t_{1}}+\sum_{j=1}^{n-1} \int_{t_{j}}^{t_{j+1}}+\int_{t_{n}}^{t_{n+1}}\right)\left(t_{n+1}-s\right)^{\alpha-1}\left(g(s)-P_{1}(s)\right) d s \\
& =I_{1}+I_{2}+I_{3} .
\end{aligned}
$$

For $I_{1}$, we have, by Assumption 1 ,

$$
\begin{aligned}
I_{1} & =\frac{1}{\Gamma(\alpha)}\left|\int_{0}^{t_{1}}\left(t_{n+1}-s\right)^{\alpha-1}\left[\frac{s-t_{1}}{-t_{1}} \int_{0}^{s} g^{\prime}(\tau) d \tau-\frac{s}{t_{1}} \int_{s}^{t_{1}} g^{\prime}(\tau) d \tau\right] d s\right| \\
& \leq C \int_{0}^{t_{1}}\left(t_{n+1}-s\right)^{\alpha-1} s^{\sigma} d s+C \int_{0}^{t_{1}}\left(t_{n+1}-s\right)^{\alpha-1} t_{1}^{\sigma} d s .
\end{aligned}
$$

Note that there exists a constant $c>0$ such that

$$
t_{n+1} \geq t_{n+1}-t_{1} \geq c t_{n+1}, n=1,2, \ldots, N-1,
$$

which follows from

$$
1 \leq \frac{t_{n+1}}{t_{n+1}-t_{1}}=\frac{\left(\frac{n+1}{N}\right)^{r}}{\left(\frac{n+1}{N}\right)^{r}-\left(\frac{1}{N}\right)^{r}}=1+\frac{1}{(n+1)^{r}-1} \leq 1+\frac{1}{2^{r}-1} \leq C .
$$

If $0<\alpha \leq 1$, then we have

$$
\begin{aligned}
\left|I_{1}\right| & \leq C\left(t_{n+1}-t_{1}\right)^{\alpha-1} \int_{0}^{t_{1}} s^{\sigma} d s+C\left(t_{n+1}-t_{1}\right)^{\alpha-1}\left(t_{1}\right)^{\sigma+1} \\
& \leq C\left(t_{n+1}-t_{1}\right)^{\alpha-1}\left(t_{1}\right)^{\sigma+1} \leq C\left(t_{n+1}\right)^{\alpha-1}\left(t_{1}\right)^{\sigma+1} \\
& \leq C\left(t_{n}\right)^{\alpha-1}\left(t_{1}\right)^{\sigma+1}=C\left(n^{r(\alpha-1)} N^{-r(\alpha+\sigma)}\right) \leq C N^{-r(\alpha+\sigma)} .
\end{aligned}
$$


If $\alpha>1$, then we have

$$
\begin{aligned}
\left|I_{1}\right| & \leq C\left(t_{n+1}\right)^{\alpha-1} \int_{0}^{t_{1}} s^{\sigma} d s+C\left(t_{n+1}\right)^{\alpha-1}\left(t_{1}\right)^{\sigma+1} \\
& \leq C\left(t_{n+1}\right)^{\alpha-1}\left(t_{1}\right)^{\sigma+1} \leq C\left(t_{n}\right)^{\alpha-1}\left(t_{1}\right)^{\sigma+1} \\
& =C\left(n^{r(\alpha-1)} N^{-r(\alpha+\sigma)}\right) \leq C N^{-r(1+\sigma)} .
\end{aligned}
$$

For $I_{2}$, we have, with $\xi_{j} \in\left(t_{j}, t_{j+1}\right), j=1,2, \ldots, n-1$ and $n=2,3, \ldots, N-1$,

$$
\left|I_{2}\right|=\left|\sum_{j=1}^{n-1} \int_{t_{j}}^{t_{j+1}}\left(t_{n+1}-s\right)^{\alpha-1} g^{\prime \prime}\left(\xi_{j}\right)\left(s-t_{j}\right)\left(s-t_{j+1}\right) d s\right| .
$$

By Assumption 1 and utilizing Stynes et al. [22, Section 5.2], with $n \geq 4$, we have

$$
\begin{aligned}
\left|I_{2}\right| \leq C\left|\sum_{j=1}^{n-1}\left(t_{j+1}-t_{j}\right)^{2}\left(t_{j}\right)^{\sigma-2} \int_{t_{j}}^{t_{j+1}}\left(t_{n+1}-s\right)^{\alpha-1} d s\right| \\
\leq C\left|\sum_{j=1}^{\left\lceil\frac{n-1}{2}\right\rceil-1}\left(t_{j+1}-t_{j}\right)^{2}\left(t_{j}\right)^{\sigma-2} \int_{t_{j}}^{t_{j+1}}\left(t_{n+1}-s\right)^{\alpha-1} d s\right| \\
\quad+C\left|\sum_{j=\left\lceil\frac{n-1}{2}\right\rceil}^{n-1}\left(t_{j+1}-t_{j}\right)^{2}\left(t_{j}\right)^{\sigma-2} \int_{t_{j}}^{t_{j+1}}\left(t_{n+1}-s\right)^{\alpha-1} d s\right| \\
=I_{21}+I_{22},
\end{aligned}
$$

where $\left\lceil\frac{n-1}{2}\right\rceil$ is the smallest integer $\geq \frac{n-1}{2}$.

For $I_{21}$, we first consider the case $0<\alpha \leq 1$, we have, with $n \geq 4$,

$$
\begin{aligned}
I_{21} & \leq C \sum_{j=1}^{\left\lceil\frac{n-1}{2}\right\rceil-1}\left(t_{j+1}-t_{j}\right)^{2}\left(t_{j}\right)^{\sigma-2}\left(t_{n+1}-t_{j+1}\right)^{\alpha-1}\left(t_{j+1}-t_{j}\right) \\
& \leq C \sum_{j=1}^{\left\lceil\frac{n-1}{2}\right\rceil-1}\left(t_{j+1}-t_{j}\right)^{3}\left(t_{j}\right)^{\sigma-2}\left(t_{n+1}-t_{j+1}\right)^{\alpha-1} .
\end{aligned}
$$

Note that, with $\xi_{j} \in[j, j+1], j=1,2, \ldots,\left\lceil\frac{n-1}{2}\right\rceil-1$,

$$
t_{j+1}-t_{j}=\left((j+1)^{r}-j^{r}\right) N^{-r}=r \xi_{j}^{r-1} N^{-r} \leq r(j+1)^{r-1} N^{-r} \leq C j^{r-1} N^{-r},
$$

and

$$
\begin{aligned}
\left(t_{n+1}-t_{j+1}\right)^{\alpha-1} & =\left(\frac{N^{r}}{(n+1)^{r}-(j+1)^{r}}\right)^{1-\alpha} \leq\left(\frac{N^{r}}{(n+1)^{r}-\left\lceil\frac{n+1}{2}\right\rceil^{r}}\right)^{1-\alpha} \\
& \leq C\left(N^{r}(n+1)^{-r}\right)^{1-\alpha} \leq C(N / n)^{r(1-\alpha)} .
\end{aligned}
$$


Thus, with $n \geq 4$,

$$
\begin{aligned}
I_{21} & \leq C \sum_{j=1}^{\left\lceil\frac{n-1}{2}\right\rceil-1}\left(j^{r-1} N^{-r}\right)^{3}(j / N)^{r(\sigma-2)}(N / n)^{r(1-\alpha)} \\
& =C \sum_{j=1}^{\left\lceil\frac{n-1}{2}\right\rceil-1} j^{r(\alpha+\sigma)-3} N^{-r(\sigma+\alpha)}(j / n)^{r(1-\alpha)}=C N^{-r(\sigma+\alpha)} \sum_{j=1}^{\left\lceil\frac{n-1}{2}\right\rceil-1} j^{r(\alpha+\sigma)-3} .
\end{aligned}
$$

Case 1 , if $r(\sigma+\alpha)<2$, we have

$$
I_{21} \leq C N^{-r(\sigma+\alpha)} \sum_{j=1}^{\left\lceil\frac{n-1}{2}\right\rceil-1} j^{r(\sigma+\alpha)-3} \leq C N^{-r(\sigma+\alpha)} .
$$

Case 2, if $r(\sigma+\alpha)=2$, we have

$$
I_{21} \leq C N^{-2} \sum_{j=1}^{\left\lceil\frac{n-1}{2}\right\rceil-1} j^{-1} \leq C N^{-2}\left(1+\frac{1}{2}+\cdots+\frac{1}{N}\right) \leq C N^{-2} \ln (N) .
$$

Case 3 , if $r(\sigma+\alpha)>2$, we have

$I_{21} \leq C N^{-r(\sigma+\alpha)} \sum_{j=1}^{\left\lceil\frac{n-1}{2}\right\rceil-1} j^{r(\sigma+\alpha)-3} \leq C N^{-r(\sigma+\alpha)} n^{r(\sigma+\alpha)-2}=C(n / N)^{r(\sigma+\alpha)-2} N^{-2} \leq C N^{-2}$.

Thus, we have, with $0<\alpha \leq 1$,

$$
I_{21} \leq\left\{\begin{array}{cl}
C N^{-r(\sigma+\alpha)}, & \text { if } r(\sigma+\alpha)<2 \\
C N^{-2} \ln (N), & \text { if } r(\sigma+\alpha)=2 \\
C N^{-2}, & \text { if } r(\sigma+\alpha)>2
\end{array}\right.
$$

We next consider the case $\alpha>1$, we have, with $n \geq 4$,

$$
\begin{aligned}
I_{21} & \leq C \sum_{j=1}^{\left\lceil\frac{n-1}{2}\right\rceil-1}\left(t_{j+1}-t_{j}\right)^{2}\left(t_{j}\right)^{\sigma-2}\left(t_{n+1}-t_{j}\right)^{\alpha-1}\left(t_{j+1}-t_{j}\right) \\
& \leq C \sum_{j=1}^{\left\lceil\frac{n-1}{2}\right\rceil-1}\left(t_{j+1}-t_{j}\right)^{3}\left(t_{j}\right)^{\sigma-2}\left(t_{n+1}\right)^{\alpha-1} \\
& \leq C \sum_{j=1}^{\left\lceil\frac{n-1}{2}\right\rceil-1}\left(j^{r-1} N^{-r}\right)^{3}(j / N)^{r(\sigma-2)}(n / N)^{r(\alpha-1)} \\
& \leq C N^{-r-r \sigma} \sum_{j=1}^{\left\lceil\frac{n-1}{2}\right\rceil-1} j^{r(1+\sigma)-3} .
\end{aligned}
$$


Thus, we have, with $\alpha>1$,

$$
I_{21} \leq\left\{\begin{array}{cl}
C N^{-r(1+\sigma)}, & \text { if } r(1+\sigma)<2, \\
C N^{-2} \ln (N), & \text { if } r(1+\sigma)=2, \\
C N^{-2}, & \text { if } r(1+\sigma)>2 .
\end{array}\right.
$$

For $I_{22}$, by (2.3) and noting that, with $\left\lceil\frac{n-1}{2}\right\rceil \leq j \leq n-1, n \geq 2$,

$$
\left(t_{j}\right)^{\sigma-2}=(j / N)^{r(\sigma-2)}=(N / j)^{r(2-\sigma)} \leq C(N / n)^{r(2-\sigma)},
$$

we have

$$
\begin{aligned}
I_{22} & \leq C\left|\sum_{j=\left\lceil\frac{n-1}{2}\right\rceil}^{n-1}\left(n^{r-1} N^{-r}\right)^{2}(N / n)^{r(2-\sigma)} \int_{t_{j}}^{t_{j+1}}\left(t_{n+1}-s\right)^{\alpha-1} d s\right| \\
& \leq C n^{r \sigma-2} N^{-r \sigma} \int_{t_{\left\lceil\frac{n-1}{2}\right\rceil} t_{n}}^{t}\left(t_{n+1}-s\right)^{\alpha-1} d s .
\end{aligned}
$$

Note that

$$
\begin{aligned}
& \int_{t_{\left\lceil\frac{n-1}{2}\right\rceil}}^{t_{n}}\left(t_{n+1}-s\right)^{\alpha-1} d s=\frac{1}{\alpha}\left[\left(t_{n+1}-t_{\left\lceil\frac{n-1}{2}\right\rceil}\right)^{\alpha}-\left(t_{n+1}-t_{n}\right)^{\alpha}\right] \\
\leq & \frac{1}{\alpha}\left(t_{n+1}-t_{\left\lceil\frac{n-1}{2}\right\rceil}\right)^{\alpha} \leq \frac{1}{\alpha}\left(t_{n+1}\right)^{\alpha}=\frac{1}{\alpha}((n+1) / N)^{r \alpha} \leq C(n / N)^{r \alpha},
\end{aligned}
$$

we get, with $n \geq 2$ and $\alpha>0$,

$I_{22} \leq C n^{r \sigma-2} N^{-r \sigma}(n / N)^{r \alpha}=C N^{-r(\sigma+\alpha)} n^{r(\sigma+\alpha)-2} \leq\left\{\begin{array}{cl}C N^{-r(\sigma+\alpha)}, & \text { if } r(\sigma+\alpha)<2, \\ C N^{-2}, & \text { if } r(\sigma+\alpha) \geq 2 .\end{array}\right.$

For $I_{3}$, we have, with $\xi_{n} \in\left(t_{n}, t_{n+1}\right), n=1,2, \ldots, N-1$,

$$
\begin{aligned}
\left|I_{3}\right| & =\left|\int_{t_{n}}^{t_{n+1}}\left(t_{n+1}-s\right)^{\alpha-1}\left(g(s)-P_{1}(s)\right) d s\right| \\
& =\left|\int_{t_{n}}^{t_{n+1}}\left(t_{n+1}-s\right)^{\alpha-1} g^{\prime \prime}\left(\xi_{n}\right)\left(s-t_{n}\right)\left(s-t_{n+1}\right) d s\right| .
\end{aligned}
$$

By Assumption 1 and (2.3), we have, with $\alpha>0$,

$$
\begin{aligned}
\left|I_{3}\right| & \leq C\left(t_{n+1}-t_{n}\right)^{2}\left(t_{n}\right)^{\sigma-2} \int_{t_{n}}^{t_{n+1}}\left(t_{n+1}-s\right)^{\alpha-1} d s \\
& =\text { eqnarray } C\left(t_{n+1}-t_{n}\right)^{2}\left(t_{n}\right)^{\sigma-2} \frac{1}{\alpha}\left(t_{n+1}-t_{n}\right)^{\alpha}=C\left(t_{n+1}-t_{n}\right)^{2+\alpha}\left(t_{n}\right)^{\sigma-2} \\
& \leq C\left(n^{r-1} N^{r}\right)^{2+\alpha}(n / N)^{r(\sigma-2)}=C n^{r(\alpha+\sigma)-2-\alpha} N^{-r(\alpha+\sigma)} \\
& \leq\left\{\begin{array}{cl}
C N^{-r(\sigma+\alpha)}, & \text { if } r(\sigma+\alpha)<2+\alpha, \\
C N^{-(2+\alpha)}, & \text { if } r(\sigma+\alpha) \geq 2+\alpha .
\end{array}\right.
\end{aligned}
$$

Obviously the bound for $I_{3}$ is stronger than the bound for $I_{21}$. 
Together these estimates complete the proof of Lemma 2.1.

Lemma 2.2 Let $\alpha>0$. We have

1. $a_{j, n+1}>0, j=0,1,2, \ldots, n+1$ where $a_{j, n+1}$ are the weights defined in (1.8).

2. $b_{j, n+1}>0, j=0,1,2, \ldots, n$, where $b_{j, n+1}$ are the weights defined in (1.7).

Proof It is obvious that $a_{0, n+1}>0, a_{n+1, n+1}>0$. For $j=1,2, \ldots, n$, we have

$$
a_{j, n+1}=\int_{t_{j-1}}^{t_{j}}\left(t_{n+1}-s\right)^{\alpha-1} \frac{s-t_{j-1}}{t_{j}-t_{j-1}} d s+\int_{t_{j}}^{t_{j+1}}\left(t_{n+1}-s\right)^{\alpha-1} \frac{s-t_{j+1}}{t_{j}-t_{j+1}} d s,
$$

which is also positive obviously. Further, we have, with $j=0,1,2, \ldots, n$,

$$
b_{j, n+1}=\frac{N^{-r \alpha}}{\alpha}\left(\left((n+1)^{r}-j^{r}\right)^{\alpha}-\left((n+1)^{r}-(j+1)^{r}\right)^{\alpha}\right)>0 .
$$

The proof of Lemma 2.2 is complete.

Lemma 2.3 Let $\alpha>0$. We have, with $n=0,1,2, \ldots, N-1$,

$$
a_{n+1, n+1} \leq C N^{-r \alpha} n^{(r-1) \alpha},
$$

where $a_{n+1, n+1}$ is defined in (1.8).

Proof We have, by (1.8), with $\xi_{n} \in(n, n+1)$,

$$
\begin{aligned}
a_{n+1, n+1} & \leq C\left(t_{n+1}-t_{n}\right)^{\alpha}=C N^{-r \alpha}\left((n+1)^{r}-n^{r}\right)^{\alpha}=C N^{-r \alpha}\left(r \xi_{n}^{r-1}\right)^{\alpha} \\
& \leq C N^{-r \alpha}\left(r(n+1)^{r-1}\right)^{\alpha} \leq C N^{-r \alpha} n^{(r-1) \alpha} .
\end{aligned}
$$

The proof of Lemma 2.3 is complete.

Lemma 2.4 Let $\alpha>0$. Assume that $g(t)$ satisfies Assumption 1 .

1. If $0<\alpha \leq 1$, we have

$$
\left|a_{n+1, n+1} \int_{0}^{t_{n+1}}\left(t_{n+1}-s\right)^{\alpha-1}\left(g(s)-P_{0}(s)\right) d s\right| \leq\left\{\begin{array}{cl}
C N^{-r(\alpha+\sigma)}, & \text { if } r(\alpha+\sigma)<1+\alpha, \\
C N^{-r(\alpha+\sigma)} \ln (N), & \text { if } r(\alpha+\sigma)=1+\alpha, \\
C N^{-1-\alpha}, & \text { if } r(\alpha+\sigma)>1+\alpha .
\end{array}\right.
$$

2. If $\alpha>1$, we have

$$
\left|a_{n+1, n+1} \int_{0}^{t_{n+1}}\left(t_{n+1}-s\right)^{\alpha-1}\left(g(s)-P_{0}(s)\right) d s\right| \leq\left\{\begin{array}{cl}
C N^{-r(\alpha+\sigma)}, & \text { if } r(\alpha+\sigma)<1+\alpha, \\
C N^{-1-\alpha}, & \text { if } r(\alpha+\sigma) \geq 1+\alpha,
\end{array}\right.
$$

where $P_{0}(s)$ is the piecewise constant function defined by, with $j=0,1,2, \ldots, n$,

$$
P_{0}(s)=g\left(t_{j}\right), \quad s \in\left[t_{j}, t_{j+1}\right] .
$$


Proof The proof is similar to the proof of Lemma 2.1. Note that

$$
\begin{aligned}
& a_{n+1, n+1} \int_{0}^{t_{n+1}}\left(t_{n+1}-s\right)^{\alpha-1}\left(g(s)-P_{0}(s)\right) d s \\
& =a_{n+1, n+1}\left(\int_{0}^{t_{1}}+\sum_{j=1}^{n-1} \int_{t_{j}}^{t_{j+1}}+\int_{t_{n}}^{t_{n+1}}\right)\left(t_{n+1}-s\right)^{\alpha-1}\left(g(s)-P_{0}(s)\right) d s \\
& =I_{1}^{\prime}+I_{2}^{\prime}+I_{3}^{\prime} .
\end{aligned}
$$

For $I_{1}^{\prime}$, we have, by Assumption 1 and Lemma 2.3

$$
\begin{aligned}
\left|I_{1}^{\prime}\right| & \leq a_{n+1, n+1}\left(\int_{0}^{t_{1}}\left(t_{n+1}-s\right)^{\alpha-1}|g(s)| d s+\int_{0}^{t_{1}}\left(t_{n+1}-s\right)^{\alpha-1}\left|P_{0}(s)\right| d s\right) \\
& \leq\left(C N^{-r \alpha} n^{(r-1) \alpha}\right)\left(\int_{0}^{t_{1}}\left(t_{n+1}-s\right)^{\alpha-1} s^{\sigma} d s+\int_{0}^{t_{1}}\left(t_{n+1}-s\right)^{\alpha-1} 0^{\sigma} d s\right) \\
& =\left(C N^{-r \alpha} n^{(r-1) \alpha}\right) \int_{0}^{t_{1}}\left(t_{n+1}-s\right)^{\alpha-1} s^{\sigma} d s .
\end{aligned}
$$

If $0<\alpha \leq 1$, by (2.1), we have

$$
\begin{aligned}
\left|I_{1}^{\prime}\right| & \leq\left(C N^{-r \alpha} n^{(r-1) \alpha}\right)\left(t_{n+1}-t_{1}\right)^{\alpha-1}\left(t_{1}\right)^{\sigma+1} \\
& \leq\left(C N^{-r \alpha} n^{(r-1) \alpha}\right)\left(C N^{-r(\alpha+\sigma)}\right)=C(n / N)^{r \alpha} n^{-\alpha}\left(C N^{-r(\alpha+\sigma)}\right) \leq C N^{-r(\alpha+\sigma)} .
\end{aligned}
$$

If $\alpha>1$, by (2.2), we have

$$
\begin{aligned}
\left|I_{1}^{\prime}\right| & \leq\left(C N^{-r \alpha} n^{(r-1) \alpha}\right)\left(t_{n+1}\right)^{\alpha-1}\left(t_{1}\right)^{\sigma+1} \\
& \leq\left(C N^{-r \alpha} n^{(r-1) \alpha}\right)\left(C N^{-r(1+\sigma)}\right)=C(n / N)^{(r-1) \alpha} N^{-\alpha} N^{-r(1+\sigma)} \\
& \leq C N^{-r(1+\sigma)-\alpha} \leq C N^{-1-\alpha} .
\end{aligned}
$$

For $I_{2}^{\prime}$, we have, with $\xi_{j} \in\left(t_{j}, t_{j+1}\right), j=1,2, \ldots, n-1$,

$$
\left|I_{2}^{\prime}\right| \leq a_{n+1, n+1} \sum_{j=1}^{n-1} \int_{t_{j}}^{t_{j+1}}\left(t_{n+1}-s\right)^{\alpha-1}\left|f^{\prime}\left(\xi_{j}\right)\right|\left(s-t_{j}\right) d s .
$$

Hence, by Assumption 1,

$$
\begin{aligned}
\left|I_{2}^{\prime}\right| & \leq C a_{n+1, n+1}\left(\sum_{j=1}^{\left\lceil\frac{n-1}{2}\right\rceil-1}+\sum_{j=\left\lceil\frac{n-1}{2}\right\rceil}^{n-1}\right)\left(t_{j+1}-t_{j}\right)\left(t_{j}\right)^{\sigma-1} \int_{t_{j}}^{t_{j+1}}\left(t_{n+1}-s\right)^{\alpha-1} d s \\
& =I_{21}^{\prime}+I_{22}^{\prime} .
\end{aligned}
$$


For $I_{21}^{\prime}$, if $0<\alpha \leq 1$, then we have, by Lemma 2.3, (2.3), (2.4), with $n \geq 4$,

$$
\begin{aligned}
I_{21}^{\prime} & \leq\left(C N^{-r \alpha} n^{(r-1) \alpha}\right) \sum_{j=1}^{\left\lceil\frac{n-1}{2}\right\rceil-1}\left(\left(t_{j+1}-t_{j}\right)^{2}\left(t_{j}\right)^{\sigma-1}\left(t_{n+1}-t_{j+1}\right)^{\alpha-1}\right) \\
& \leq\left(C N^{-r \alpha} n^{(r-1) \alpha}\right) \sum_{j=1}^{\left\lceil\frac{n-1}{2}\right\rceil-1}\left(j^{r-1} N^{-r}\right)^{2}(j / N)^{r(\sigma-1)}(N / n)^{r(1-\alpha)} \\
& =C(n / N)^{r \alpha} \sum_{j=1}^{\left\lceil\frac{n-1}{2}\right\rceil-1} j^{r(\alpha+\sigma)-2-\alpha}(j / n)^{\alpha}(j / n)^{r(1-\alpha)} N^{-r(\alpha+\sigma)} \\
& \leq C N^{-r(\alpha+\sigma)} \sum_{j=1}^{\left\lceil\frac{n-1}{2}\right\rceil-1} j^{r(\alpha+\sigma)-2-\alpha} \leq\left\{\begin{array}{cc}
l l C N^{-r(\alpha+\sigma)}, & \text { if } r(\alpha+\sigma)<1+\alpha, \\
C N^{-r(\alpha+\sigma)} \ln (N), & \text { if } r(\alpha+\sigma)=1+\alpha, \\
C N^{-1-\alpha}, & \text { if } r(\alpha+\sigma)>1+\alpha .
\end{array}\right.
\end{aligned}
$$

If $\alpha>1$, we have

$$
\begin{aligned}
I_{21}^{\prime} & \leq\left(C N^{-r \alpha} n^{(r-1) \alpha}\right) \sum_{j=1}^{\left\lceil\frac{n-1}{2}\right\rceil-1}\left(\left(t_{j+1}-t_{j}\right)^{2}\left(t_{j}\right)^{\sigma-1}\left(t_{n+1}\right)^{\alpha-1}\right) \\
& \leq\left(C N^{-r \alpha} n^{(r-1) \alpha}\right) \sum_{j=1}^{\left\lceil\frac{n-1}{2}\right\rceil-1}\left(j^{r-1} N^{-r}\right)^{2}(j / N)^{r(\sigma-1)}(N / n)^{r(1-\alpha)} \\
& =C(n / N)^{(r-1) \alpha} N^{-\alpha} N^{-r \sigma-r} \sum_{j=1}^{\left\lceil\frac{n-1}{2}\right\rceil-1} j^{r+r \sigma-2} \\
& \leq C N^{-\alpha-r \sigma-r} \sum_{j=1}^{\left\lceil\frac{n-1}{2}\right\rceil-1} j^{r+r \sigma-2} .
\end{aligned}
$$

Note that $r+r \sigma-2>-1$ for any $r \geq 1$. Hence, we have

$$
I_{21}^{\prime} \leq C N^{-\alpha-r \sigma-r} n^{r+r \sigma-1}=C(n / N)^{r+r \sigma-1} N^{-1-\alpha} \leq C N^{-1-\alpha} .
$$

For $I_{22}^{\prime}$, we have

$$
I_{22}^{\prime} \leq\left(C N^{-r \alpha} n^{(r-1) \alpha}\right) \sum_{j=\left\lceil\frac{n-1}{2}\right\rceil}^{n-1}\left(\left(t_{j+1}-t_{j}\right)\left(t_{j}\right)^{\sigma-1} \int_{t_{j}}^{t_{j+1}}\left(t_{n+1}-s\right)^{\alpha-1} d s\right) .
$$

By (2.3) and noting that, with $\left\lceil\frac{n-1}{2}\right\rceil \leq j \leq n-1, n \geq 2$,

$$
\left(t_{j}\right)^{\sigma-1}=(j / N)^{r(\sigma-1)}=(N / j)^{r(1-\sigma)} \leq C(N / n)^{r(1-\sigma)},
$$


we have, by (2.5), with $\alpha>0$,

$$
\begin{aligned}
I_{22}^{\prime} & \leq\left(C N^{-r \alpha} n^{(r-1) \alpha}\right) \sum_{j=\left\lceil\frac{n-1}{2}\right\rceil}^{n-1}\left(\left(C n^{r-1} N^{-r}\right)(N / n)^{r(1-\sigma)} \int_{t_{j}}^{t_{j+1}}\left(t_{n+1}-s\right)^{\alpha-1} d s\right) \\
& \leq\left(C N^{-r \alpha} n^{(r-1) \alpha}\right) n^{r-1-r+\sigma} N^{-r+r-r \sigma}(n / N)^{r \alpha} \leq C n^{r(\sigma+\alpha)-1-\alpha} N^{-r(\sigma+\alpha)} \\
& \leq\left\{\begin{array}{cl}
C N^{-r(\sigma+\alpha)}, & \text { if } r(\sigma+\alpha)<1+\alpha, \\
C N^{-1-\alpha}, & \text { if } r(\sigma+\alpha) \geq 1+\alpha .
\end{array}\right.
\end{aligned}
$$

For $I_{3}^{\prime}$, we have, with $\alpha>0$,

$$
\begin{aligned}
\left|I_{3}^{\prime}\right| & \leq\left(C N^{-r \alpha} n^{(r-1) \alpha}\right)\left(t_{n+1}-t_{n}\right)\left(t_{n}\right)^{\sigma-1}\left(t_{n+1}-t_{n}\right)^{\alpha} \\
& \leq\left(C N^{-r \alpha} n^{(r-1) \alpha}\right)\left(t_{n+1}-t_{n}\right)^{1+\alpha}\left(t_{n}\right)^{\sigma-1} .
\end{aligned}
$$

By (2.3), we have

$$
\begin{aligned}
\left|I_{3}^{\prime}\right| & \leq\left(C N^{-r \alpha} n^{(r-1) \alpha}\right)\left(n^{r-1} N^{-r}\right)^{1+\alpha}(n / N)^{r(\sigma-1)} \\
& =C(n / N)^{r \alpha} n^{-\alpha} n^{r(\alpha+\sigma)-\alpha-1} N^{-r(\alpha+\sigma)} \\
& \leq C n^{r(\alpha+\sigma)-\alpha-1} N^{-r(\alpha+\sigma)} \\
& \leq\left\{\begin{array}{cl}
C N^{-r(\alpha+\sigma)}, & \text { if } r(\alpha+\sigma)<1+\alpha, \\
C N^{-1-\alpha}, & \text { if } r(\alpha+\sigma) \geq 1+\alpha .
\end{array}\right.
\end{aligned}
$$

Together these estimates complete the proof of Lemma 2.4 .

Lemma 2.5 Let $\alpha>0$. There exists a positive constant $C$ such that

$$
\begin{aligned}
& \sum_{j=0}^{n} a_{j, n+1} \leq C T^{\alpha}, \\
& \sum_{j=0}^{n} b_{j, n+1} \leq C T^{\alpha},
\end{aligned}
$$

where $\alpha_{j, n+1}$ and $b_{j, n+1}, j=0,1,2, \ldots, n$ are defined by (1.8) and (1.7), respectively.

Proof We only prove (2.6). The proof of (2.7) is similar. Note that

$$
\int_{0}^{t_{n+1}}\left(t_{n+1}-s\right)^{\alpha-1} g(s) d s=\sum_{j=0}^{n+1} a_{j, n+1} g\left(t_{j}\right)+R_{1},
$$

where $R_{1}$ is the remainder term. Let $g(s)=1$, we have

$$
\sum_{j=0}^{n+1} a_{j, n+1}=\int_{0}^{t_{n+1}}\left(t_{n+1}-s\right)^{\alpha-1} \cdot 1 d s=\frac{1}{\alpha}\left(t_{n+1}\right)^{\alpha} \leq C T^{\alpha} .
$$

Thus, (2.6) follows by the fact $a_{n+1, n+1}>0$ in Lemma 2.2.

Now we turn to the proof of Theorem 1.4. 
Proof of Theorem 1.4 Subtracting (1.3) from (1.6), we have

$$
\begin{aligned}
y\left(t_{n+1}\right)-y_{n+1} & \\
= & \frac{1}{\Gamma(\alpha)}\left\{\int_{0}^{t_{n+1}}\left(t_{n+1}-s\right)^{\alpha-1}\left(f(s, y(s))-P_{1}(s)\right) d s\right. \\
& \left.+\sum_{j=0}^{n} a_{j, n+1}\left(f\left(t_{j}, y\left(t_{j}\right)\right)-f\left(t_{j}, y_{j}\right)\right)+a_{n+1, n+1}\left(f\left(t_{n+1}, y\left(t_{n+1}\right)\right)-f\left(t_{n+1}, y_{n+1}^{P}\right)\right)\right\} \\
= & \frac{1}{\Gamma(\alpha)}(I+I I+I I I) .
\end{aligned}
$$

The term $I$ is estimated by Lemma 2.1. For $I I$, we have, by Lemma 2.2 and the Lipschitz condition of $f$,

$$
\begin{aligned}
|I I| & =\left|\sum_{j=0}^{n} a_{j, n+1}\left(f\left(t_{j}, y\left(t_{j}\right)\right)-f\left(t_{j}, y_{j}\right)\right)\right| \leq \sum_{j=0}^{n} a_{j, n+1}\left|f\left(t_{j}, y\left(t_{j}\right)\right)-f\left(t_{j}, y_{j}\right)\right| \\
& \leq L \sum_{j=0}^{n} a_{j, n+1}\left|y\left(t_{j}\right)-y_{j}\right| .
\end{aligned}
$$

For $I I I$, we have, by Lemma 2.2 and the Lipschitz condition for $f$,

$$
|I I I|=\left|a_{n+1, n+1}\left(f\left(t_{n+1}, y\left(t_{n+1}\right)\right)-f\left(t_{n+1}, y_{n+1}^{P}\right)\right)\right| \leq a_{n+1, n+1} L\left|y\left(t_{n+1}\right)-y_{n+1}^{P}\right| .
$$

Note that

$$
\begin{aligned}
y\left(t_{n+1}\right)-y_{n+1}^{P} & =\frac{1}{\Gamma(\alpha)}\left\{\int_{0}^{t_{n+1}}\left(t_{n+1}-s\right)^{\alpha-1}\left(f(s, y(s))-P_{0}(s)\right) d s\right. \\
& \left.+\sum_{j=0}^{n} b_{j, n+1}\left(f\left(t_{j}, y\left(t_{j}\right)\right)-f\left(t_{j}, y_{j}\right)\right)\right\} .
\end{aligned}
$$

Thus,

$$
\begin{aligned}
|I I I| \leq C & a_{n+1, n+1} L \int_{0}^{t_{n+1}}\left(t_{n+1}-s\right)^{\alpha-1}\left|f(s, y(s))-P_{0}(s)\right| d s \\
& +C a_{n+1, n+1} L \sum_{j=0}^{n} b_{j, n+1}\left|f\left(t_{j}, y\left(t_{j}\right)\right)-f\left(t_{j}, y_{j}\right)\right| \\
& =I I I_{1}+I I I_{2} .
\end{aligned}
$$

The term $I I I_{1}$ is estimated by Lemma 2.4. For $I I I_{2}$, we have, by Lemmas 2.2, 2.3,

$$
\begin{aligned}
I I I_{2} & \leq C a_{n+1, n+1} \sum_{j=0}^{n} b_{j, n+1}\left|y\left(t_{j}\right)-y_{j}\right| \leq\left(C N^{-r \alpha} n^{(r-1) \alpha}\right) \sum_{j=0}^{n} b_{j, n+1}\left|y\left(t_{j}\right)-y_{j}\right| \\
& \leq C(n / N)^{(r-1) \alpha} N^{-\alpha} \sum_{j=0}^{n} b_{j, n+1}\left|y\left(t_{j}\right)-y_{j}\right| \leq C N^{-\alpha} \sum_{j=0}^{n} b_{j, n+1}\left|y\left(t_{j}\right)-y_{j}\right| .
\end{aligned}
$$


Hence, we obtain

$$
\begin{aligned}
& \left|y\left(t_{n+1}\right)-y_{n+1}\right| \leq C|I|+C \sum_{j=0}^{n} a_{j, n+1}\left|y\left(t_{j}\right)-y_{j}\right| \\
& +C\left|I I I_{1}\right|+C N^{-\alpha} \sum_{j=0}^{n} b_{j, n+1}\left|y\left(t_{j}\right)-y_{j}\right| .
\end{aligned}
$$

To complete the proof of Theorem 1.4, we shall use the mathematical induction.

We first consider the case $0<\alpha \leq 1$. In this case, we discuss the error estimates in the following four cases.

Case 1. Let $r(\alpha+\sigma)>\max \{2,1+\alpha\}=2$. Assume that there exists a constant $C_{0}>0$ such that, with $j=0,1,2, \ldots, n, n=0,1,2, \ldots, N-1$,

$$
\left|y\left(t_{j}\right)-y_{j}\right| \leq C_{0} N^{-1-\alpha} \text {, }
$$

we shall show that

$$
\left|y\left(t_{n+1}\right)-y_{n+1}\right| \leq C_{0} N^{-1-\alpha} .
$$

In fact, by Lemmas 2.1 and 2.4, we have

$$
\begin{aligned}
\left|y\left(t_{n+1}\right)-y_{n+1}\right| & \leq C N^{-2}+C \sum_{j=0}^{n} a_{j, n+1}\left|y\left(t_{j}\right)-y_{j}\right| \\
& +C N^{-1-\alpha}+C N^{-\alpha} \sum_{j=0}^{n} b_{j, n+1}\left|y\left(t_{j}\right)-y_{j}\right| \\
& \leq C N^{-2}+C_{0} C T^{\alpha} N^{-1-\alpha}+C N^{-1-\alpha}+T^{\alpha} C_{0} N^{-\alpha} N^{-1-\alpha} .
\end{aligned}
$$

Following the idea of the proof for [8, Lemma 3.1, pp.41], we may first choose $T$ sufficiently small such that the second term of the right hand side of (2.9) is less than $\frac{C_{0}}{2} N^{-1-\alpha}$, then choose $N$ sufficiently large and $C_{0}$ sufficiently large such that the summation of the other terms in the right hand side of (2.9) is also less than $\frac{C_{0}}{2} N^{-1-\alpha}$. Thus, we get

$$
\left|y\left(t_{n+1}\right)-y_{n+1}\right| \leq C_{0} N^{-1-\alpha} .
$$

Case 2. Let $r(\alpha+\sigma) \leq \min \{2,1+\alpha\}=1+\alpha$. Assume that there exists a constant $C_{0}>0$ such that, with $j=0,1,2, \ldots, n, n=0,1,2, \ldots, N-1$,

$$
\left|y\left(t_{j}\right)-y_{j}\right| \leq C_{0} N^{-r(\alpha+\sigma)} \text {. }
$$

Following the similar argument as in Case 1, we may show that

$$
\left|y\left(t_{n+1}\right)-y_{n+1}\right| \leq C_{0} N^{-r(\alpha+\sigma)} .
$$

Case 3. Let $1+\alpha<r(\alpha+\sigma) \leq 2$. We may show that

$$
\left|y\left(t_{n+1}\right)-y_{n+1}\right| \leq C_{0} N^{-1-\alpha} .
$$

Case 4. Let $r(\alpha+\sigma)=1+\alpha$. We may show that

$$
\left|y\left(t_{n+1}\right)-y_{n+1}\right| \leq C_{0} N^{-1-\alpha} \ln (N) .
$$


We next consider the case $\alpha>1$. In this case, we also discuss the error estimates in the following four cases.

Case 1. Let $r>\max \left\{\frac{1+\alpha}{\alpha+\sigma}, \frac{2}{1+\sigma}\right\}=\frac{2}{1+\sigma}$. Assume that there exists a constant $C_{0}>0$ such that, with $j=0,1,2, \ldots, n, n=0,1,2, \ldots, N-1$,

$$
\left|y\left(t_{j}\right)-y_{j}\right| \leq C_{0} N^{-2},
$$

we shall show that

$$
\left|y\left(t_{n+1}\right)-y_{n+1}\right| \leq C_{0} N^{-2} .
$$

In fact, we have, using the same argument as in the proof of (2.8),

$$
\begin{aligned}
\left|y\left(t_{n+1}\right)-y_{n+1}\right| & \leq C N^{-2}+C \sum_{j=0}^{n} a_{j, n+1}\left|y\left(t_{j}\right)-y_{j}\right| \\
& +C N^{-1-\alpha}+C N^{-\alpha} \sum_{j=0}^{n} b_{j, n+1}\left|y\left(t_{j}\right)-y_{j}\right| \\
& \leq C N^{-2}+C_{0} T^{\alpha} N^{-2}+C N^{-1-\alpha}+T^{\alpha} C_{0} N^{-\alpha} N^{-2} .
\end{aligned}
$$

Following the idea of the proof for [8, Lemma 3.1, pp.41], we may first choose $T$ sufficiently small such that the second term of the right hand side of (2.10) is less than $\frac{C_{0}}{2} N^{-2}$, then choose $N$ sufficiently large and $C_{0}$ sufficiently large such that the summation of the other terms in the right hand side of (2.10) is also less than $\frac{C_{0}}{2} N^{-2}$. Thus, we get

$$
\left|y\left(t_{n+1}\right)-y_{n+1}\right| \leq C_{0} N^{-2} .
$$

Case 2. Let $r<\min \left\{\frac{1+\alpha}{\alpha+\sigma}, \frac{2}{1+\sigma}\right\}=\frac{1+\alpha}{\alpha+\sigma}$. Assume that there exists a constant $C_{0}>0$ such that, with $j=0,1,2, \ldots, n, n=0,1,2, \ldots, N-1$,

$$
\left|y\left(t_{j}\right)-y_{j}\right| \leq C_{0} N^{-r(1+\sigma)} \text {. }
$$

Following the similar argument as in Case 1, we may show that

$$
\left|y\left(t_{n+1}\right)-y_{n+1}\right| \leq C_{0} N^{-r(1+\sigma)} .
$$

Case 3. Let $\frac{1+\alpha}{\alpha+\sigma} \leq r<\frac{2}{1+\sigma}$. We may show that

$$
\left|y\left(t_{n+1}\right)-y_{n+1}\right| \leq C_{0} N^{-r(1+\sigma)} .
$$

Case 4. Let $r=\frac{2}{1+\sigma}$. We may show that

$$
\left|y\left(t_{n+1}\right)-y_{n+1}\right| \leq C_{0} N^{-2} \ln (N) .
$$

Together these estimates complete the proof of Theorem 1.4. 
Table 1 Maximum nodal errors and orders of convergence for Example 3.1 with $\alpha=0.8$ and $\beta=0.9$

\begin{tabular}{llllll}
\hline & $N=40$ & $N=80$ & $N=160$ & $N=320$ & $N=640$ \\
\hline$r=1$ & $1.43 \mathrm{E}-2$ & $7.68 \mathrm{E}-3$ & $4.12 \mathrm{E}-3$ & $2.21 \mathrm{E}-3$ & $1.18 \mathrm{E}-3$ \\
$r=\frac{1+\alpha}{\beta}$ & 0.897 & 0.899 & 0.899 & 0.899 & \\
& $5.18 \mathrm{E}-4$ & $1.49 \mathrm{E}-4$ & $4.27 \mathrm{E}-5$ & $1.23 \mathrm{E}-5$ & $3.51 \mathrm{E}-6$ \\
& 1.800 & 1.800 & 1.800 & 1.800 & \\
\hline
\end{tabular}

\section{Numerical examples}

In this section, we will give some numerical examples to illustrate the convergence orders of the numerical method (1.6) under the different smoothness assumptions of ${ }_{0}^{C} D_{t}^{\alpha} y$ in (1.3). For simplicity, we only present the numerical results for the case $\alpha \in(0,1)$. Similarly, we may obtain the numerical results for $\alpha>1$.

Example 3.1 Consider, with $0<\alpha<1,0<\beta<1$ and $\alpha<\beta$,

$$
\begin{aligned}
& { }_{0}^{C} D_{t}^{\alpha} y(t)=\frac{\Gamma(1+\beta)}{\Gamma(1+\beta-\alpha)} t^{\beta-\alpha}+t^{2 \beta}-y(t)^{2}, \quad t \in(0, T], \\
& y(0)=y_{0},
\end{aligned}
$$

where $y_{0}=0$, and the exact solution is $y(t)=t^{\beta}$, and ${ }_{0}^{C} D_{t}^{\alpha} y(t)=\frac{\Gamma(1+\beta)}{\Gamma(1+\beta-\alpha)} t^{\beta-\alpha}$, which implies that the regularity of ${ }_{0}^{C} D_{t}^{\alpha} y(t)$ behaves as $t^{\beta-\alpha}$. Thus, we see that ${ }_{0}^{C} D_{t}^{\alpha} y(t)$ satisfies the Assumption 1.

Let $N$ be a positive integer. Let $0=t_{0}<t_{1}<\cdots<t_{N}=T$ be the graded meshes on $[0, T]$, where $t_{j}=T(j / N)^{r}, j=0,1,2, \ldots, N$ with $r \geq 1$. For simplicity, we choose $T=1$. Assume that $y\left(t_{j}\right)$ and $y_{j}, j=0,1,2, \ldots N$ are the solutions of (1.3) and (1.6), respectively. We have, by Theorem 1.4 with $\sigma=\beta-\alpha$,

$$
\left\|e_{N}\right\|:=\max _{0 \leq j \leq N}\left|y\left(t_{j}\right)-y_{j}\right| \leq\left\{\begin{array}{cl}
C N^{-r \beta}, & \text { if } r<\frac{1+\alpha}{\beta}, \\
C N^{-r \beta} \ln (N), & \text { if } r=\frac{1+\alpha}{\beta} \\
C N^{-(1+\alpha)}, & \text { if } r>\frac{1+\alpha}{\beta} .
\end{array}\right.
$$

Table 2 Maximum nodal errors and orders of convergence for Example 3.1 with $\alpha=0.6$ and $\beta=0.9$

\begin{tabular}{llllll}
\hline & $N=40$ & $N=80$ & $N=160$ & $N=320$ & $N=640$ \\
\hline$r=1$ & $8.95 \mathrm{E}-3$ & $4.83 \mathrm{E}-3$ & $2.60 \mathrm{E}-3$ & $1.39 \mathrm{E}-3$ & $7.46 \mathrm{E}-4$ \\
$r=\frac{1+\alpha}{\beta}$ & 0.889 & 0.896 & 0.898 & 0.899 & \\
& $1.29 \mathrm{E}-3$ & $3.88 \mathrm{E}-4$ & $1.20 \mathrm{E}-4$ & $3.82 \mathrm{E}-5$ & $1.23 \mathrm{E}-5$ \\
& 1.727 & 1.687 & 1.655 & 1.635 & \\
\hline
\end{tabular}


Table 3 Maximum nodal errors and orders of convergence for Example 3.1 with $\alpha=0.4$ and $\beta=0.9$

\begin{tabular}{llllll}
\hline & $N=40$ & $N=80$ & $N=160$ & $N=320$ & $N=640$ \\
\hline$r=1$ & $4.33 \mathrm{E}-3$ & $2.40 \mathrm{E}-3$ & $1.30 \mathrm{E}-3$ & $7.01 \mathrm{E}-4$ & $3.76 \mathrm{E}-4$ \\
$r=\frac{1+\alpha}{\beta}$ & 0.853 & 0.881 & 0.892 & 0.897 & \\
& $4.64 \mathrm{E}-3$ & $1.46 \mathrm{E}-3$ & $4.84 \mathrm{E}-4$ & $1.66 \mathrm{E}-4$ & $5.87 \mathrm{E}-5$ \\
& 1.667 & 1.595 & 1.541 & 1.503 & \\
\hline
\end{tabular}

For the different $\alpha \in(0,1)$, we choose the different $r$ and the different $N=20 \times$ $2^{l}, l=1,2,3,4,5$. We obtain the maximum nodal errors $\left\|e_{N}\right\|_{\infty}$ defined above with respect to the different $N$. We also calculate the experimental order of convergence (EOC) by $\log 2\left(\frac{\left\|e_{N}\right\|_{\infty}}{\left\|e_{2 N}\right\|_{\infty}}\right)$.

In Tables 1, 2 and 3, we choose $\beta=0.9$ and we obtain the experimental orders of convergence (EOC) and the maximum nodal errors with respect to the different $N$. We see that the experimental orders of convergence (EOC) are almost $O\left(N^{-r \beta}\right)=$ $O\left(N^{-(1+\alpha)}\right.$ if we choose $r=\frac{1+\alpha}{\beta}$.

Example 3.2 Consider, with $0<\alpha<1$,

$$
\begin{aligned}
& { }_{0}^{C} D_{t}^{\alpha} y(t)+y(t)=0, \quad t \in(0, T], \\
& y(0)=y_{0},
\end{aligned}
$$

where $y_{0}=1$. The exact solution is $y(t)=E_{\alpha, 1}\left(-t^{\alpha}\right)$, and ${ }_{0}^{C} D_{t}^{\alpha} y(t)=$ $-E_{\alpha, 1}\left(-t^{\alpha}\right)$, where $E_{\alpha, \gamma}(z)$ is the Mittag-Leffler function defined by

$$
E_{\alpha, \gamma}(z)=\sum_{k=0}^{\infty} \frac{z^{k}}{\Gamma(\alpha k+\gamma)}, \quad \alpha, \gamma>0
$$

Hence, we have

$$
{ }_{0}^{C} D_{t}^{\alpha} y(t)=-1-\frac{\left(-t^{\alpha}\right)}{\Gamma(\alpha+1)}-\frac{\left(-t^{\alpha}\right)^{2}}{\Gamma(2 \alpha+1)}-\ldots,
$$

Table 4 Maximum nodal errors and orders of convergence for Example 3.2 with $\alpha=0.8$

\begin{tabular}{llllll}
\hline & $N=40$ & $N=80$ & $N=160$ & $N=320$ & $N=640$ \\
\hline$r=1$ & $1.14 \mathrm{E}-4$ & $4.43 \mathrm{E}-5$ & $1.59 \mathrm{E}-5$ & $5.47 \mathrm{E}-6$ & $1.85 \mathrm{E}-6$ \\
$r=\frac{1+\alpha}{2 \alpha}$ & 1.370 & 1.481 & 1.535 & 1.564 & \\
& $9.48 \mathrm{E}-5$ & $2.75 \mathrm{E}-5$ & $8.05 \mathrm{E}-6$ & $2.36 \mathrm{E}-6$ & $6.94 \mathrm{E}-7$ \\
& 1.784 & 1.773 & 1.768 & 1.767 & \\
\hline
\end{tabular}


Table 5 Maximum nodal errors and orders of convergence for Example 3.2 with $\alpha=0.6$

\begin{tabular}{llllll}
\hline & $N=40$ & $N=80$ & $N=160$ & $N=320$ & $N=640$ \\
\hline$r=1$ & $7.57 \mathrm{E}-4$ & $4.34 \mathrm{E}-4$ & $2.20 \mathrm{E}-4$ & $1.05 \mathrm{E}-4$ & $4.83 \mathrm{E}-5$ \\
& 0.803 & 0.981 & 1.069 & 1.118 & \\
$r=\frac{1+\alpha}{2 \alpha}$ & $2.58 \mathrm{E}-4$ & $9.66 \mathrm{E}-5$ & $3.41 \mathrm{E}-5$ & $1.17 \mathrm{E}-5$ & $3.93 \mathrm{E}-6$ \\
& 1.418 & 1.503 & 1.547 & 1.570 & \\
\hline
\end{tabular}

which implies that the regularity of ${ }_{0}^{C} D_{t}^{\alpha} y(t)$ behaves as $c+c t^{\alpha}, 0<\alpha<1$. By Theorem 1.4 with $\sigma=\alpha$, we have

$$
\left\|e_{N}\right\|_{\infty}:=\max _{0 \leq j \leq N}\left|y\left(t_{j}\right)-y_{j}\right| \leq\left\{\begin{array}{cl}
C N^{-r(2 \alpha)}, & \text { if } r<\frac{1+\alpha}{2 \alpha} \\
C N^{-r(2 \alpha)} \ln (N), & \text { if } r=\frac{1+\alpha}{2 \alpha} \\
C N^{-(1+\alpha)}, & \text { if } r>\frac{1+\alpha}{2 \alpha}
\end{array}\right.
$$

In Tables 4, 5 and 6, we obtain the experimental orders of convergence (EOC) and the maximum nodal errors with respect to the different $N$. We see that the experimental orders of convergence (EOC) are almost $O\left(N^{-r(2 \alpha)}\right)=O\left(N^{-(1+\alpha)}\right.$ if we choose $r=\frac{1+\alpha}{\alpha}$.

Remark 3.1 In the numerical examples above, the choices of the mesh grading parameters are based on the fact that we know certain properties of the exact solution. But in a realistic application, these properties are usually not known. Therefore, it might be better to motivate the choice of $r$ by some properties of the given data.

In the applications, in some cases, we may determine the $\sigma$ in Assumption 1 by using the given properties of $f$, then we may decide the value of $r$ by Theorem 1.5. For example, by Remark 1.2, if we know $f \in C^{m}(G), m \geq 2$ and $\alpha \in(0,1)$, then we see that $\sigma=\alpha$. We will consider how to choose $r$ based on some general properties of the given data in our future research.

Table 6 Maximum nodal errors and orders of convergence for Example 3.2 with $\alpha=0.4$

\begin{tabular}{lcclll}
\hline & $N=40$ & $N=80$ & $N=160$ & $N=320$ & $N=640$ \\
\hline$r=1$ & $5.12 \mathrm{E}-4$ & $8.33 \mathrm{E}-4$ & $1.00 \mathrm{E}-3$ & $8.17 \mathrm{E}-4$ & $5.78 \mathrm{E}-4$ \\
& -0.702 & -0.268 & 0.296 & 0.498 & \\
$r=\frac{1+\alpha}{2 \alpha}$ & $2.58 \mathrm{E}-4$ & $9.66 \mathrm{E}-5$ & $3.41 \mathrm{E}-5$ & $1.17 \mathrm{E}-5$ & $3.93 \mathrm{E}-6$ \\
& 1.123 & 1.233 & 1.304 & 1.343 & \\
\hline
\end{tabular}


Acknowledgments The work of the first author was carried out during her stay at the University of Chester, which is supported financially by Shanxi province government, P. R. China. She thanks the Department of Mathematics, University of Chester, for its warm hospitality and providing a very good working condition for her during her stay in Chester.

Open Access This article is distributed under the terms of the Creative Commons Attribution 4.0 International License (http://creativecommons.org/licenses/by/4.0/), which permits unrestricted use, distribution, and reproduction in any medium, provided you give appropriate credit to the original author(s) and the source, provide a link to the Creative Commons license, and indicate if changes were made.

\section{References}

1. Cao, J., Xu, C.: A high order schema for the numerical solution of the fractional ordinary differential equations. J. Comput. Phys. 238, 154-168 (2013)

2. Deng, W.H.: Short memory principle and a predictor-corrector approach for fractional differential equations. J. Comput. Appl. Math. 206, 1768-1777 (2007)

3. Deng, W.H.: Numerical algorithm for the time fractional Fokker-Planck equation. J. Comput. Phys. 227, 1510-1522 (2007)

4. Diethelm, K.: Generalized compound quadrature formulae for finite-part integral. IMA J. Numer. Anal. 17, 479-493 (1997)

5. Diethelm, K.: The analysis of fractional differential equations, an application-oriented exposition using differential operators of caputo type. Lecture Notes in Mathematics, Springer, Berlin (2010)

6. Diethelm, K.: Efficient solutions of multi-term fractional differential equations using $P(E C)^{m} E$ methods. Computing 71, 305-319 (2003)

7. Diethelm, K., Ford, N.J.: Analysis of fractional differential equations. J. Math. Anal. Appl. 265(2), 229-248 (2002)

8. Diethelm, K., Ford, N.J., Freed, A.D.: Detailed error analysis for a fractional Adams method. Numer. Algor. 36, 31-52 (2004)

9. Diethelm, K., Ford, N.J., Freed, A.D.: A predictor-corrector approach for the numerical solution of fractional differential equations. Nonlinear Dyn. 29, 3-22 (2002)

10. Kilbas, A.A., Srivastava, H.M., Trujillo, J.J.: Theory and applications of fractional differential equations. Elsevier, Amsterdam (2006)

11. Kolk, M., Pedas, A., Tamme, E.: Modified spline collocation for linear fractional differential equations. J. Comput. Appl. Math. 283, 28-40 (2015)

12. Li, C., Yi, Q., Chen, A.: Finite difference methods with non-uniform meshes for nonlinear fractional differential equations. J. Comput. Phys. 316, 614-631 (2016)

13. Li, Z., Yan, Y., Ford, N.J.: Error estimates of a high order numerical method for solving linear fractional differential equation. Appl. Numer. Math. 114, 201-220 (2017)

14. Li, C., Zeng, F.: The finite difference methods for fractional ordinary differential equations. Numer. Funct. Anal. Optim. 34, 149-179 (2013)

15. Lubich, C.: Runge-Kutta theory for Volterra and Abel integral equations of the second kind. Math. Comp. 41, 87-102 (1983)

16. Oldham, K., Spanier, J.: The fractional calculus. Academic Press, San Diego (1974)

17. Pal, K., Liu, F., Yan, Y.: Numerical solutions for fractional differential equations by extrapolation. Lect. Notes Comput. Sci. Springer Ser. 9045, 299-306 (2015)

18. Pedas, A., Tamme, E.: Numerical solution of nonlinear fractional differential equations by spline collocation methods. J. Comput. Appl. Math. 255, 216-230 (2014)

19. Quintana-Murillo, J., Yuste, S.B.: A finite difference method with non-uniform timesteps for fractional diffusion and diffusion-wave equations. Eur. Phys. J. Spec. Top. 222, 1987-1998 (2013)

20. Podlubny, I.: Fractional differential, equations, mathematics in science and engineering, vol. 198. Academic Press, Cambridge (1999)

21. Stynes, M.: Too much regularity may force too much uniqueness. Fractional Calc. Appl. Anal. 19, $1554-1562(2016)$

22. Stynes, M., O'riordan, E., Gracia, J.L.: Error analysis of a finite difference method on graded meshes for a time-fractional diffusion equation. SIAM J. Numer. Anal. 55, 1057-1079 (2017) 
23. Yan, Y., Pal, K., Ford, N.J.: Higher order numerical methods for solving fractional differential equations. BIT Numer. Math. 54, 555-584 (2014)

24. Yuste, S.B., Quintana-Murillo, J.: Fast, accurate and robust adaptive finite difference methods for fractional diffusion equations. Numer. Algor. 71, 207-228 (2016)

25. Zhang, Y., Sun, Z., Liao, H.: Finite difference methods for the time fractional diffusion equation on non-uniform meshes. J. Comput. Phys. 265, 195-210 (2014)

26. Zhao, L., Deng, W.H.: Jacobian-predictor-corrector approach for fractional ordinary differential equations. Adv. Comput. Math. 40, 137-165 (2014) 